\title{
The Use of Binary Quantization for the Acquisition of Low SNR Ultrasonic Signals: A Study of the Input Dynamic Range
}

\author{
Julio Isla and Frederic Celga
}

\begin{abstract}
Low-power excitation and/or low sensitivity transducers, such as electromagnetic acoustic transducers, piezoelectric paints, air-coupled transducers, and small elements of dense arrays, may produce signals below the noise threshold at the receiver. The information from those noisy signals can be recovered after averaging or pulse compression using binary (1-b) quantization only without experiencing significant losses. Hence, no analog-to-digital converter is required, which reduces the data throughput and makes the electronics faster, more compact, and energy efficient. All these are especially attractive for applications that require arrays with many channels and high sampling rates, where the sampling rate can be as high as the system clock. In this paper, the theory of binary quantization is reviewed, mainly from previous work on wireless sensor networks, and the signal-to-noise ratio (SNR) of the input signals under which binary quantization is of practical interest for ultrasound applications is investigated. The main findings are that in most practical cases binary quantization can be used with small errors when the input SNR is on the order of $8 \mathrm{~dB}$ or less. Moreover, the maximum SNR after binary quantization and averaging can be estimated as $10 \log _{10} N-2 \mathrm{~dB}$, where $N$ is the number of averages.
\end{abstract}

Index Terms-Analog-digital conversion (ADC), quantization, ultrasonic transducers.

\section{INTRODUCTION}

$\mathbf{M}$ ANY ultrasound applications produce signals that are weak and potentially fall below the noise level at the receiver. However, after quantization, the signal-to-noise ratio (SNR) is increased by ensemble averaging and filtering or pulse compression techniques. This is possible because the excitation signals are recurrent. Some examples of applications where received signals are below the noise threshold can be found in [1] and [2] for electromagnetic acoustic transducers, [3] for piezoelectric paints, [4] for photoacoustic imaging, [5]-[8] for air-coupled ultrasound, and [9]-[11] for guided ultrasonic waves. Several other applications exist for the inspection of highly attenuating materials such as Inconel [12] and distance and displacement measurements using ultrasound [13], [14].

In those cases, the information has been shown to be recovered using quantization levels that are not much bigger

Manuscript received April 26, 2016; accepted May 17, 2016. Date of publication May 23, 2016; date of current version September 12, 2016. The work of F. Cegla was supported by the Engineering and Physical Sciences Research Council under Grant EP/K033565/1.

The authors are with the Department of Mechanical Engineering, Imperial College London, London SW7 2AZ, U.K. (e-mail: j.isla13@imperial.ac.uk; f.cegla@imperial.ac.uk).

Digital Object Identifier 10.1109/TUFFC.2016.2571843 than the signal itself [4], [6], [10], [15]-[17]—the explanation of how this is possible was attributed to the effect of dithering [18]-[20]. Of particular interest is [6], where it was reported that the information can be recovered using binary (1-b) quantization only. The same result was reported in [21] (a decade before) where binary quantization was employed with time-reversal techniques and pulse compression without degrading the spatial or temporal resolution of an array of sensors.

These findings have an important implication in the acquisition of signals embedded in noise since no analog-to-digital converters (ADCs) are then required; standard ADCs could be replaced by a comparator and a binary latch. An example of such a system is shown in Fig. 1, where the output of each transducer or analog channel is connected to an amplifier. After the amplifier there is an antialiasing filter to remove the high-frequency components followed by a comparator, which acts as a 1-b ADC. The comparator may require a latch control signal to synchronize its output with the digital system. However, the dashed rectangle in Fig. 1 highlights that depending on the digital interface, neither the comparator nor the latch control signal may be required, and hence the analog channel could be directly connected to the digital input.

Using comparators, the size of the digital bus is greatly simplified to one digital line per channel, and therefore, data throughput is reduced. In general, without an ADC, the acquisition system becomes faster, more compact, and energy efficient. All these are especially attractive for applications that require arrays with many channels and high sampling rates, where the sampling rate can be as high as the system clock; the maximum sampling rate of standard ADCs is usually less than the system clock.

The binary quantization of noisy signals has been investigated extensively in the past years, mainly in the field of wireless sensor networks (WSNs) [22]-[24], where the motivations were also limited power and bandwidth of the acquisition and data transmission systems. It is necessary to emphasize that binary quantization is actually employed to later estimate a parameter of interest, in this case the signal embedded in noise, and not to necessarily reconstruct the exact sampled signal (see [25] for a discussion on this).

One of the main findings has been that when the signals are below the noise threshold, the difference between binary quantization and no quantization at all, i.e., using an infinite-bit ADC, is roughly only $2 \mathrm{~dB}$ [21], [22] and that 


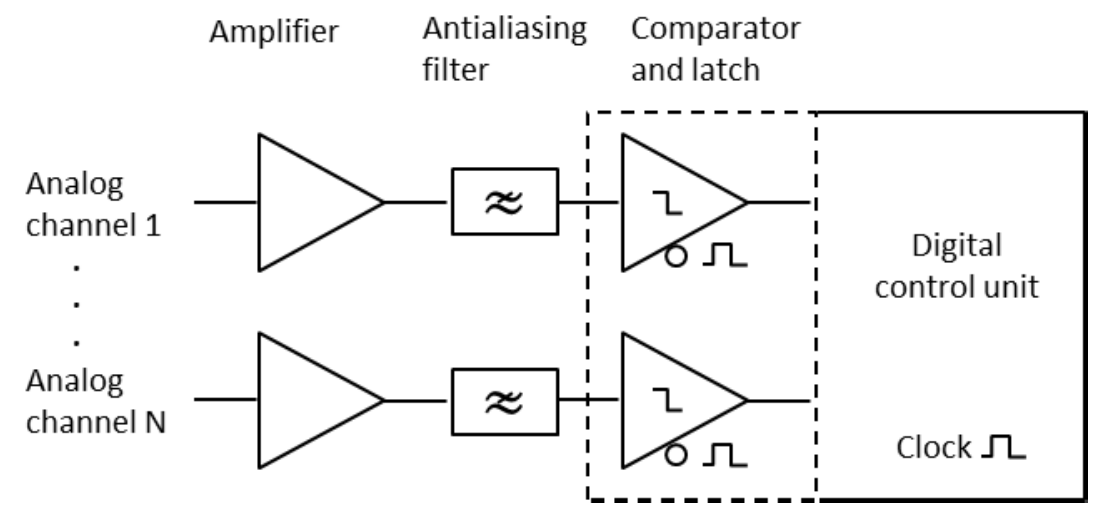

Fig. 1. N-channel binary acquisition system. The sampling frequency can be as high as the system clock frequency. External comparators and/or latches may not be necessary in some cases.

this difference increases as the SNR of the signal to be quantized increases [22], [23]. Further work has also been conducted to select the optimum threshold for the binary comparator [23], [24], [26].

For signals with greater SNR, i.e., above the noise threshold, the work has been focused on incorporating some control input before quantization or adding extra quantization levels [27]. However, this approach introduces extra complexity in the acquisition system. The main goal here is to investigate the conditions under which a simple system, as described in Fig. 1, can be employed for ultrasonic applications.

Information about the maximum input SNR range where binary quantization is of practical interest is not readily available. In this paper, we review the theory of binary quantization from previous work (mainly that related to WSNs) and then investigate the input SNR range of practical interest for ultrasound applications.

This paper is organized as follows. First, the theory related to binary quantization from previous work is presented, and then the maximum input SNRs that can be employed are investigated theoretically. Following this, some numerical simulations are carried out to corroborate the theoretical results. Experiments with binary-quantized ultrasound signals are presented, and finally, conclusions are drawn.

\section{BinARY QuAntization AND AVERAgIng}

The theory behind binary quantization has been reported in [22] and [23]. However, in this section, it is reviewed again in a way that highlights how the different sources of error affect the results. The main sources of error are: 1) the error introduced by binary quantization itself and 2) the error caused when only a limited number of quantized samples or realizations are added (averaged).

\section{A. Transfer Function of the Binary Quantizer After Averaging}

Consider a stationary random or stochastic process $Y(t)$, where $t \in \mathbb{Z}$ hereinafter, that has $N$ independent copies $Y_{1}(t), \ldots, Y_{N}(t)$, which are just time functions. Say this stochastic process represents the electrical noise introduced

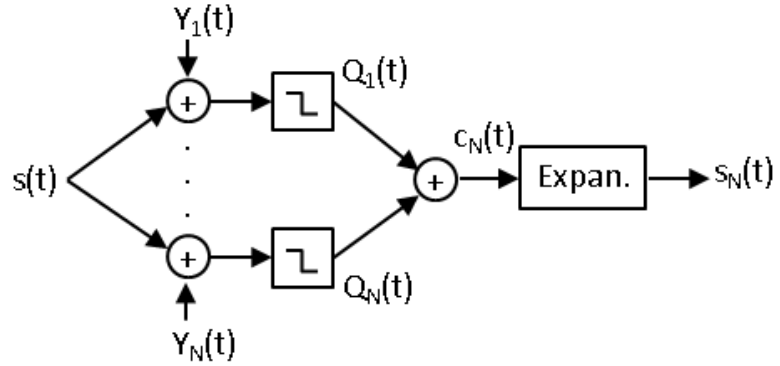

Fig. 2. Stages of binary quantization. $N$ realizations are added after the comparators, which produces an integer number $c_{N}$. The output of the quantizers has to be expanded to compensate for the nonlinear compressing behavior of $E[Q]$.

by an amplifier. At any instant $t_{i}, Y\left(t_{i}\right)$ is a random variable, whereas $Y_{n}\left(t_{i}\right)$ is just a number.

Let $s(t)$ be a deterministic signal invariant to each copy of $Y(t)$, in this case, it can be said that $s(t)$ is recurrent, and let

$$
X(t)=s(t)+Y(t) .
$$

Fig. 2 shows the addition of $s(t)$ to each of the $N$ values taken on by the copies of $Y(t)$, which are the input to binary quantizers with corresponding outputs $Q_{1}(t), \ldots, Q_{N}(t)$, where $Q(t)$ is the stochastic process that represents the output of the $N$ quantizers.

The output of the $n$ binary quantizer can take on the following values at $t_{i}$ :

$$
Q_{n}\left(t_{i}\right)= \begin{cases}1, & X_{n}\left(t_{i}\right)>0 \\ -1, & X_{n}\left(t_{i}\right) \leq 0\end{cases}
$$

To simplify the notation, we write that the expected value of $Q$ for any $t$ is

$$
E[Q]=\bar{F}_{X}(0)-F_{X}(0)
$$

where $F_{X}(x)$ is the cumulative distribution function (cdf) of $X$ (at any $t$ ) and $\bar{F}_{X}=1-F_{X} \cdot F_{X}$ is equal to the cdf of $Y$ offset by $s$. Hereinafter, $t$ can be dropped at any time to simplify the notation.

If $Y$ is assumed to be normally distributed, the following equation can be derived based on the fact that $X$ is then 


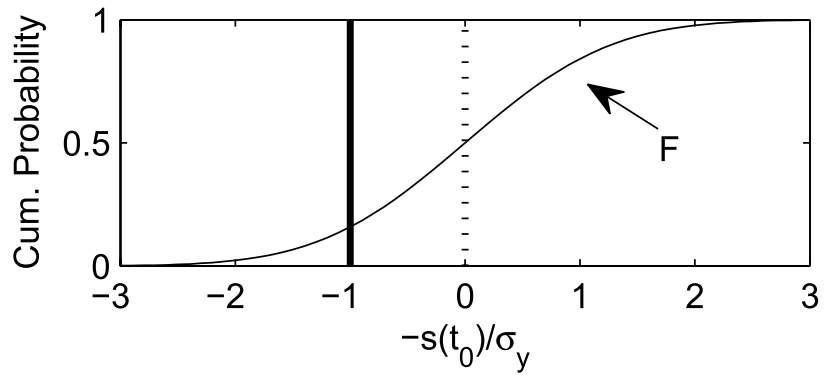

(a)

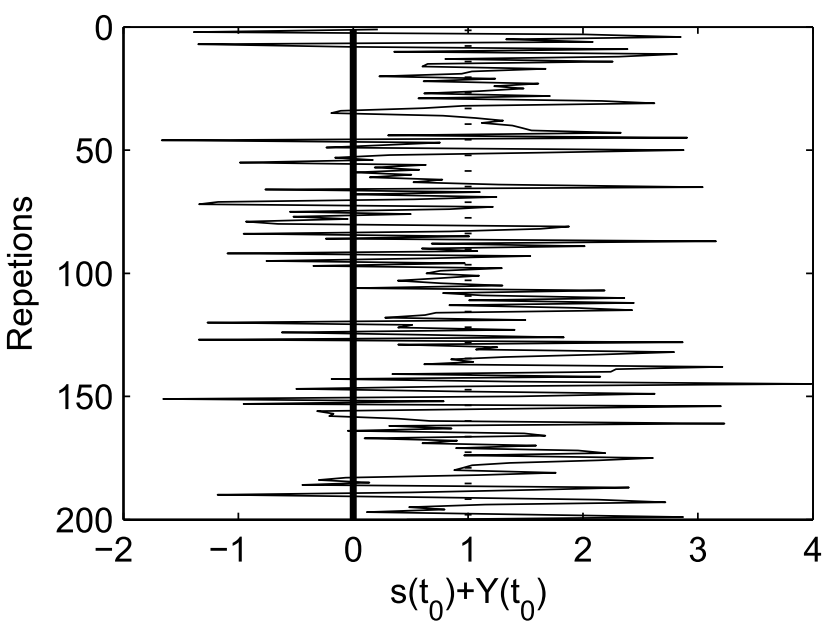

(b)

Fig. 3. (a) cdf of the standard normal distribution, $F$. (b) Repetitions of $X\left(t_{0}\right)$ with $s\left(t_{0}\right)=1$. If the number of realizations at either side of a certain level is known, then the mean value of the normal distribution can be estimated relative to its standard deviation.

normally distributed with mean $s(t)$ and standard deviation $\sigma_{y}$ :

$$
E[Q(t)]=1-2 F\left[-\frac{s(t)}{\sigma_{y}}\right]
$$

where $F$ is the cdf of the standard normal distribution (mean $\mu=0$ and standard deviation $\sigma=1$ ). In this case, $Y$ acts as a noisy carrier for the signal $s$, which is the foundation of dithering. It is interesting to highlight that when $\sigma_{y} \rightarrow 0$, $E[Q(t)] \rightarrow-1$ if $s(t)<0$; otherwise, $E[Q(t)] \rightarrow 1$.

Equation (4) can be understood intuitively based on Fig. 3(a) and (b), where $F$ is plotted together with several repetitions or realizations of $X$ at $t_{0}$ with $s\left(t_{0}\right)=1$. If the number of realizations at either side of a certain level and their distribution are known, then the mean value of the distribution can be estimated relative to its standard deviation, $\sigma_{y}$.

Let the result after adding $N$ copies of $Q$ be

$$
c_{N}(t)=\sum_{n=1}^{N} Q_{n}(t)
$$

Note $c_{N}(t) \in \mathbb{Z}$ with $c_{N}(t) \in[-N, N]$, which introduces a round-off error.

Due to $F$ being a nonlinear function, (4) describes a type of nonlinear quantization similar to that of $\mu$ - and A-law companders [28], where a compression function (4) is uniformly quantized by $2 N+1$ levels after adding $N$ copies of it. To compensate for the nonlinearity introduced by the compression function, an expansion function is required, which is basically the inverse of (4). Hence, the resulting signal is

$$
s_{N}(t)=-F^{-1}\left[\frac{N-c_{N}(t)}{2 N}\right]
$$

where $F^{-1}$ is the inverse of $F$.

Since (6) is subject to random variations any time $N$ copies of $Q$ are added, for completeness, we say that $s_{N}(t)$ is a copy of a random process $S_{N}(t)$ and then

$$
s(t) \propto E\left[S_{N}(t)\right]+e(t), \quad-N<c_{N}(t)<N
$$

where $e(t)$ is the round-off error that appears due to the fact $c_{N}(t) \in \mathbb{Z}$. It will be shown that $e(t)$ is negligible compared with the standard deviation of $S_{N}(t), \sigma_{S_{N}}$, when $N$ is large and $-N<c_{N}(t)<N$.

\section{B. Quantization Errors and SNR}

The variance after adding $N$ copies of $Q$ is (see Appendix A)

$$
\sigma_{Q, N}^{2}=4 N \cdot F\left(\frac{s}{\sigma_{y}}\right) \bar{F}\left(\frac{s}{\sigma_{y}}\right)
$$

where $\bar{F}=1-F$; note that $t$ has been dropped to simplify the notation.

Now, suppose $N$ is large and $-N<c_{N}<N$, then the standard deviation of $S_{N}$ can be approximated as (see Appendix B for the rationale behind this approximation)

$\sigma_{S_{N}} \approx \begin{cases}s_{N}+F^{-1}\left(\frac{N-c_{N}-\sigma_{Q, N}}{2 N}\right) & N>c_{N} \geq 0 \\ s_{N}-F^{-1}\left(\frac{N-c_{N}+\sigma_{Q, N}}{2 N}\right) & -N<c_{N}<0 .\end{cases}$

In [23] and [24], closed-form Cramer-Rao and Chernoff bounds are used to estimate this variance; however, the authors found that the approximation in (9) produced accurate results for all the values that were simulated.

In addition, the SNR at the output of the binary quantizer can be approximated as

$$
\mathrm{SNR} \approx \frac{E\left[S_{N}\right]}{\sigma_{S_{N}}}-N<c_{N}<N
$$

It is interesting to investigate the SNR when $s / \sigma_{y} \ll 1$. In this case, $F$ can be regarded as a linear function of $s$ [see Fig. 3(a)]. Therefore, the SNR is the same before and after the expansion operation. Then, if the round-off error $e$ in (7) is negligible, the following approximation for the SNR is obtained (see [21], [22]):

$$
\left.\mathrm{SNR}\right|_{\frac{s}{\sigma_{y}} \ll 1} \approx \frac{N \cdot E[Q]}{\sigma_{Q, N}} \approx \frac{s}{\sigma_{y}} \sqrt{\frac{2}{\pi} N} .
$$

Hence, when $\left(s / \sigma_{y}\right) \ll 1$, the resulting SNR after binary quantization and $N$ realizations added is just roughly 0.8 times $(2 \mathrm{~dB})$ smaller than without any quantization at all, i.e., an ADC that uses infinite quantization levels and produces an SNR $=\left(s / \sigma_{y}\right) \sqrt{N}$. Note that $F^{\prime}(0)=(2 / \pi)^{1 / 2}$, where $F^{\prime}$ is the derivative of $F$. 


\section{Limits of Binary Quantization}

If $c_{N} \in\{-N, N\}$, then $s_{N} \in\{-\infty, \infty\}$ even when $-\infty<$ $s<\infty$. By substituting $c_{N} \in\{-N+0.5, N-0.5\}$ in (6), the upper and lower bounds that define the quantizer range where the round-off error $e$ take on finite values are obtained

$$
-F^{-1}\left(1-\frac{1}{4 N}\right)>s_{N}>-F^{-1}\left(\frac{1}{4 N}\right) \text {. }
$$

To prevent $s_{N}$ from being infinite in the event $c_{N} \in\{-N, N\}$, $s_{N}$ can simply be truncated to the closer of these bounds; this of course introduces a significant round-off error.

The impact of $e$ on the results can be inferred by the number of times that $c_{N} \in\{-N, N\}$ occurs in $N$ realizations. Then, it is useful to find the probability of reaching the condition $c_{N}=N$ for a given $s / \sigma_{y}$. This is the probability of obtaining $Q_{n}=1$, i.e., $\bar{F}\left(-s / \sigma_{y}\right)$, for each of the $N$ realizations of $X$

$$
p_{N}=\left[\bar{F}\left(-\frac{s}{\sigma_{y}}\right)\right]^{N} \text {. }
$$

Moreover, to numerically investigate the standard deviation at the output of the quantizer for $N$ added realizations $\left(\sigma_{S_{N}}\right)$, $M$ sets with $N$ realizations each have to be assessed. The probability of having $c_{N}=N L$ times in $M$ realizations follows the binomial distribution, and hence

$$
p_{L}=\left(\begin{array}{c}
M \\
L
\end{array}\right) p_{N}^{L}\left(1-p_{N}\right)^{M-L}
$$

while the probability of having $c_{N}=N L$ or more times in $M$ realizations is the cumulative probability of having $c_{N}=N$ from $L$ to $M$ times

$$
p_{L, \text { cum }}=\sum_{k=L}^{M}\left(\begin{array}{c}
M \\
k
\end{array}\right) p_{N}^{k}\left(1-p_{N}\right)^{M-k} .
$$

Equation (15) can be used to predict, for example, the value of $s$ for which $c_{N}=N$ occurs more than $10 \%$ of the time, i.e., $L=0.1 M$, with a probability of say 0.9 . This may be used to indicate when $e$ has a significant impact on the results.

It is equally useful to know the probability of $c_{N}=N$ occurring at least once in $M$ realizations, which is equivalent to the complement of the probability of $c_{N}=N$ not occurring, i.e., $1-p_{N}$, in $M$ realizations

$$
p_{1, \text { cum }}=1-\left(1-p_{N}\right)^{M} .
$$

\section{Numerical Simulations}

A set of $10^{2}$ and $10^{4}$ samples were normally distributed with $\sigma=1$ to obtain the random variable $Y\left(t_{i}\right)$. The mean of the distribution was varied from -5 to $15 \mathrm{~dB}$ in intervals of $1 \mathrm{~dB}$ to simulate $s$. Each sample was binary quantized and then added (averaged) and expanded using (6); this process is summarized in Fig. 2. Hereinafter, for the sake of brevity, these three operations will be referred to as quantization. The maximum/minimum value of each realization after the expansion operation was limited to the upper/lower bound in (12), so that infinite results were avoided. Each step was repeated $10^{4}$ times to investigate the expected values and the SNRs at the output of the quantizer.

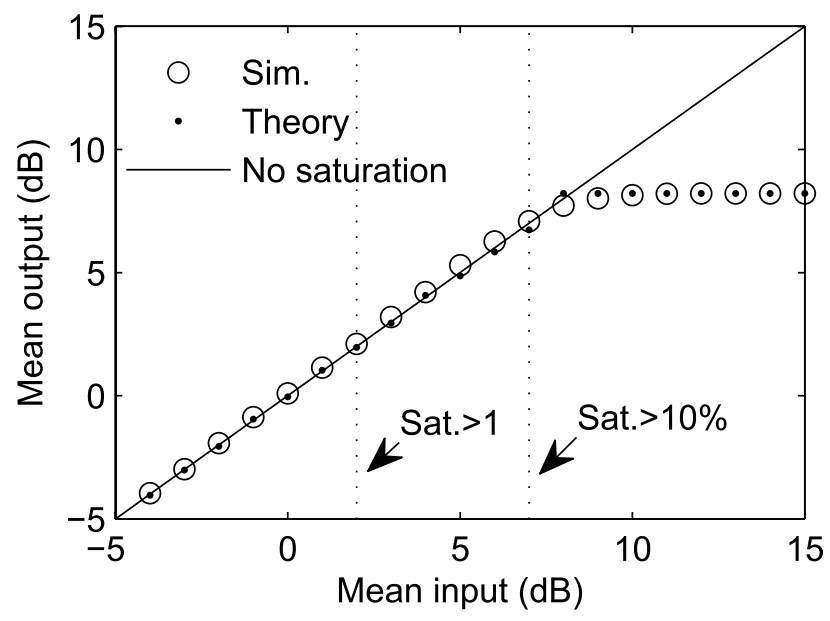

(a)

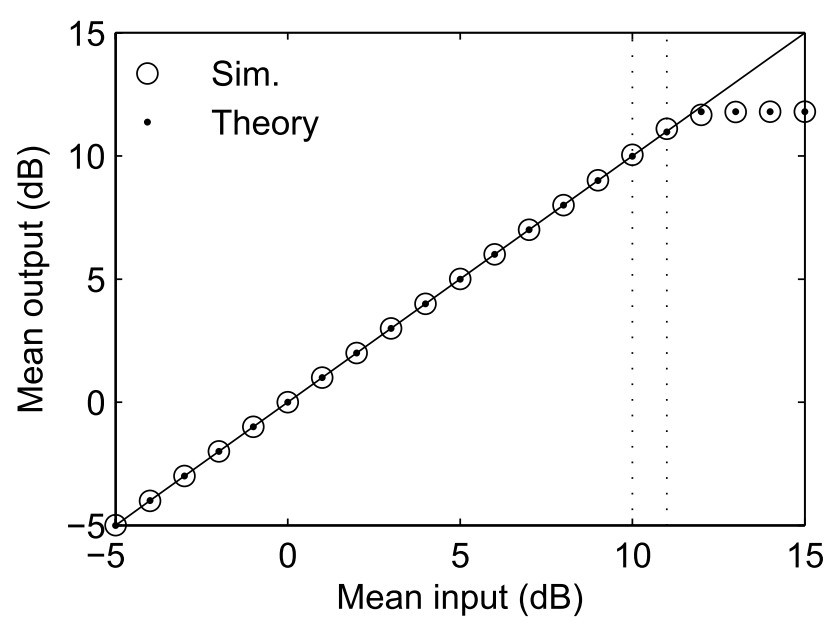

(b)

Fig. 4. Mean value before and after quantization of a normal distribution with $\sigma=1$ for $10^{4}$ sets of (a) $10^{2}$ and (b) $10^{4}$ samples. The continuous line represents the expected signal without saturation error. The vertical dotted line (Sat. > 1) indicates the occurrence of saturation at least once with a probability of $10^{-4}$. The vertical dotted line (Sat. $>10 \%$ ) indicates the occurrence of saturation $10 \%$ of the time with a probability of 0.9 .

\section{A. Expected Value at the Output of the Quantizer}

Fig. 4 shows the expected value at the output of the quantizer for different input cases. The input is the ratio between the mean and the standard deviation of the set of samples at the input; this ratio is basically the distribution mean $s$ since the standard deviation $\sigma=1$. The circle markers in Fig. 4(a) and (b) correspond to the simulated sets of $10^{2}$ and $10^{4}$ added samples, respectively. The dot markers represent the theoretical expected values according to (6). The continuous line represents the ideal acquisition process, where there is no saturation or round-off error $e$. The vertical dotted line (labeled Sat. > 1) indicates the occurrence of saturation at least once with probability of $10^{-4}$; this is basically the value of $s$ for which (16) yields $10^{-4}$. The other vertical dotted line (labeled Sat. $>10 \%$ ) indicates the occurrence of saturation $10 \%$ of the time with a probability of 0.9 ; this is the value of $s$ for which (15) gives 0.9 with $L=0.1 \mathrm{M}$.

In general, there is good agreement between the theory presented above and the simulations. It can be observed 


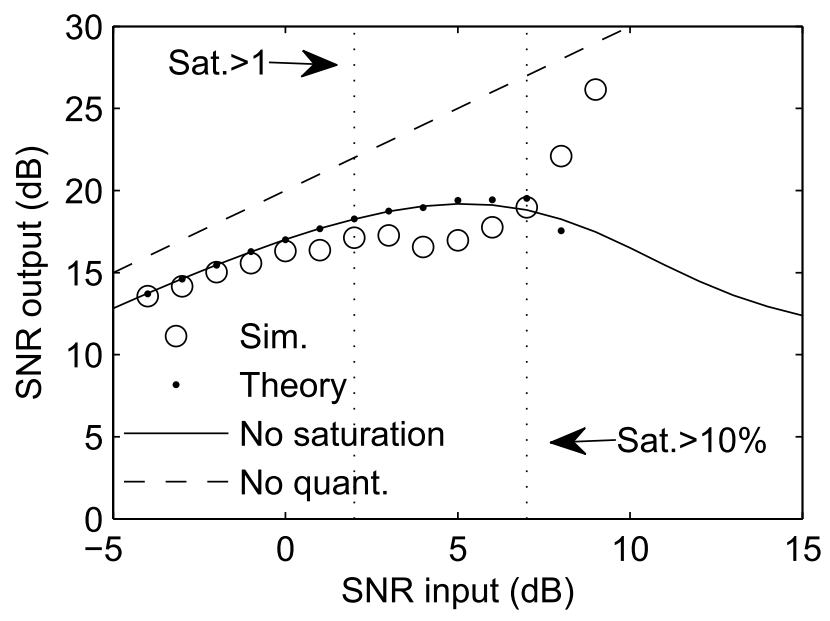

(a)

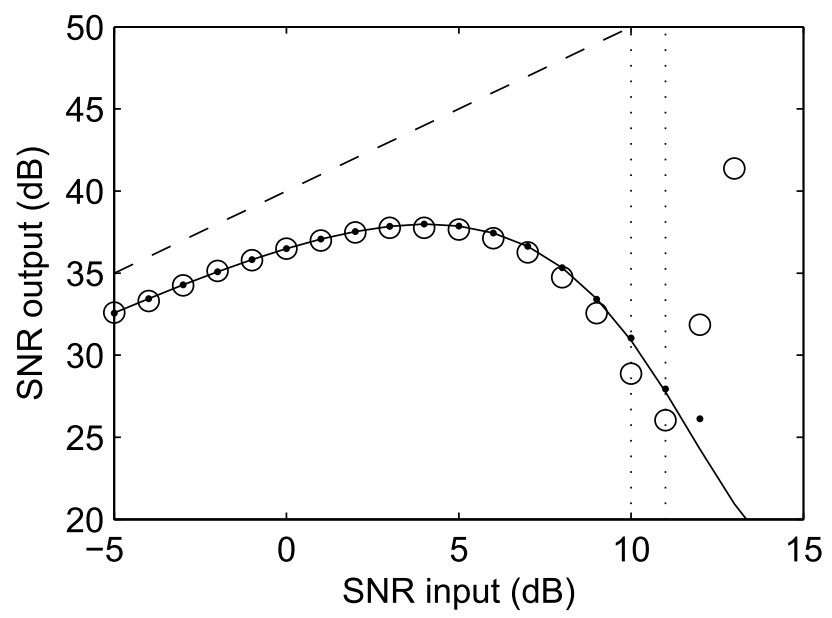

(b)

Fig. 5. SNR before and after quantization. $10^{4}$ sets of (a) $10^{2}$ and (b) $10^{4}$ realizations. The continuous line represents the expected SNR without saturation or round-off error. The vertical dotted line $($ Sat. $>1$ ) indicates the occurrence of saturation at least once with a probability of $10^{-4}$. The vertical dotted line (Sat. $>10 \%$ ) indicates the occurrence of saturation $10 \%$ of the time with a probability of 0.9 . The dashed line indicates the resulting SNR without quantization.

that (15) can be used to predict the value of the input mean where the linearity of the system changes, i.e., it becomes nonlinear. Moreover, when the maximum/minimum value of each realization is truncated using (12) so that the result is not infinite, the input range that produces a linear output is extended from the first occurrence of saturation (marked by Sat. $>1$ ) to roughly where saturation occurs $10 \%$ of the time. This increase is approximately 5 and $1 \mathrm{~dB}$ for the sets of $10^{2}$ and $10^{4}$ samples, respectively; note that truncation has a greater impact on the set with fewer samples. Overall, the greater the number of samples (equivalent in practice to the number of averages) in a set, the greater the bounds in (12), and therefore, the greater the input range of the quantizer.

\section{B. Output SNR}

Fig. 5 shows the SNR before and after quantization. The SNR of each simulation is computed as the ratio of the mean and the standard deviation of the set (circle markers). The dot

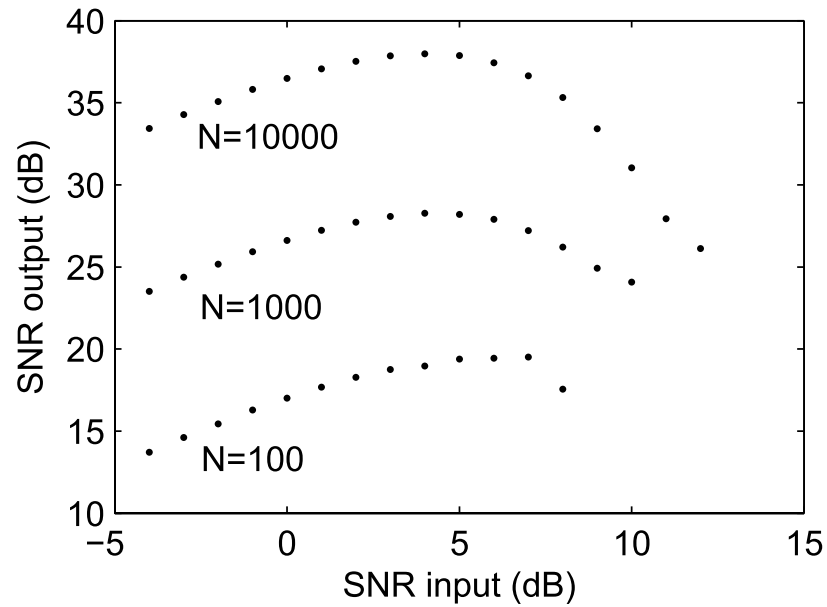

Fig. 6. Outputs of (10) for SNR inputs between -5 and $15 \mathrm{~dB}$ using $10^{4}$ sets of $10^{2}, 10^{3}$, and $10^{4}$ realizations.

markers represent the outputs of (10). The continuous line is the theoretical result assuming there is no saturation or round-off error; this is calculated by replacing $c_{N}$ by $N \cdot E[Q]$ in (6) and (9). The dashed line represents the resulting SNR without quantization, i.e., the standard deviation of the sum of all of the samples in a set. The vertical dotted lines, labeled Sat. $>1$ and Sat. $>10 \%$, are the same as in Fig. 4.

Again, the theory presented above and the simulations match well before saturation takes place (i.e., below the input SNR marked by the vertical dotted line labeled Sat. $>1$ ). This confirms that the round-off error $e$ is negligible in this interval. Note that for an input SNR below $-5 \mathrm{~dB}$, the difference between binary quantization and no quantization at all (dashed line) is roughly $2 \mathrm{~dB}$ as predicted by (11). In general, the resulting SNR after binary quantization is always smaller than the SNR without any quantization at all. The resulting SNR produced by any other type of quantization, e.g., a 2- or 12-b quantization, should lie between these two cases.

For input SNR values between the dotted lines Sat. $>1$ and Sat. $>10 \%$ saturation causes the output SNR to be overestimated by no more than $2 \mathrm{~dB}$. Note that the distance between the dotted lines shortens as the number of added samples in the set increases. The results that correspond to an input SNR beyond the line Sat. $>10 \%$ should be ignored as errors due to saturation are significant and the information is lost.

Fig. 6 shows the outputs of (10) for an input SNR between -5 and $15 \mathrm{~dB}$ using $10^{4}$ sets of $10^{2}, 10^{3}$, and $10^{4}$ realizations. It can be observed that the curves appear to be vertically offset and that the output SNR increases as a function of $N$ as long as the input $\mathrm{SNR}$ remains below $\sim 8-12 \mathrm{~dB}$. It can be noted that the maximum output $\mathrm{SNR}\left(\mathrm{SNR}_{\max }\right)$ occurs when the input SNR is roughly $4 \mathrm{~dB}$ and that for each number of samples, the corresponding $\mathrm{SNR}_{\max }$ is slightly smaller than the number of samples in a decibel scale $\left(10 \log _{10} N\right)$.

In Fig. 7, the difference $10 \log _{10} N-\mathrm{SNR}_{\max }$ is plotted with a dashed-dotted curve for in the interval $N \epsilon\left[10^{2}, 10^{6}\right]$, realizations. From the curve, the following expression can be used as a good estimate of $\mathrm{SNR}_{\max }$ when $N>10^{3}$ :

$$
\mathrm{SNR}_{\max } \approx 10 \log _{10} N-2 \quad N>10^{3} \text {. }
$$




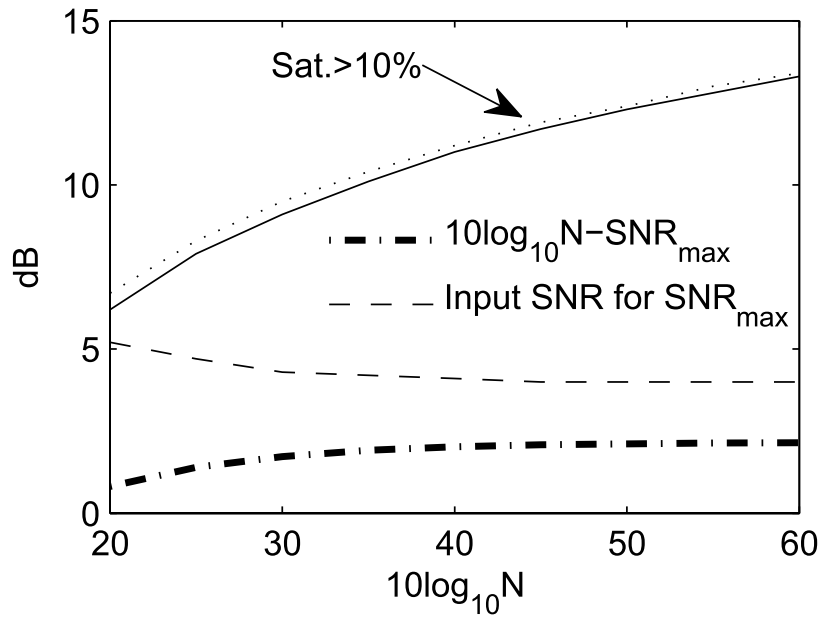

Fig. 7. Input SNR that yields $\mathrm{SNR}_{\max }$ (dashed curve), $10 \log _{10} N-\mathrm{SNR}_{\max }$ dashed-dotted curve), and input SNR where saturation occurs $10 \%$ of the time with a probability of 0.9 (labeled Sat. $>10 \%$ ) versus the number of added realizations $N$. The dotted and continuous lines labeled Sat. $>10 \%$ correspond to sets of 10 and $10^{4}$ samples, respectively.

The input SNR that yields $\mathrm{SNR}_{\max }$ is also shown in Fig. 7 (dashed-dotted curve). This confirms the previous observation that $\mathrm{SNR}_{\max }$ occurs when the input SNR is roughly $4 \mathrm{~dB}$.

Finally, the dotted and continuous curves in Fig. 7 indicate the input SNR where saturation occurs $10 \%$ of the time with a probability of 0.9 for sets of 10 and $10^{4}$ samples, respectively. Note that the size of the set has a minor effect on the results. These curves are a good approximation of the maximum input SNR that produces a nondistorted output of the quantizer. For example, when $N=10^{3}$, the maximum input SNR that produces a nondistorted output is approximately $9 \mathrm{~dB}$; the maximum input SNR increases only by roughly $4 \mathrm{~dB}$ when $N=10^{6}$. In the interval $N \in\left[10^{3}, 10^{6}\right]$, the maximum input $\mathrm{SNR}\left(\mathrm{SNR}_{\max , \text { in }}\right)$ can be approximated as

$$
\mathrm{SNR}_{\max , \text { in }} \approx \frac{4}{3} \log _{10} N+4, \quad N \in\left[10^{3}, 10^{6}\right] .
$$

Overall, a minimum bound for the input SNR range (difference between the maximum and minimum input SNRs in decibels), which can also be understood as the input signal dynamic range, can be approximated as

$$
\mathrm{D}>10 \log _{10} N-\mathrm{SNR}_{\min }+2, \quad N \in\left[10^{3}, 10^{6}\right]
$$

where $\mathrm{SNR}_{\min }$ is the minimum tolerable SNR after quantization and averaging (defined for each application beforehand). As an example, if $\mathrm{SNR}_{\min }=20 \mathrm{~dB}$ and it is desired $\mathrm{D}>8 \mathrm{~dB}$, then $N>100$. The dynamic range $\mathrm{D}$ is therefore tunable by adjusting the number of averages $N$. This means that the dynamic range can be increased at the cost of decreased measurement speed in order to suit the requirements of different applications. In general, binary quantization offers a lower input SNR range compared with standard ADCs. This is because ADCs can be thought of as a superposition of offset binary quantizers. However, once the signals are embedded in noise, the advantage of using a standard ADC is only a $2 \mathrm{~dB}$ increase in SNR. It is important to recall that filtering increases the SNR by removing the noise components outside the frequency band of interest. Therefore, the effective input SNR range is also increased by filtering.

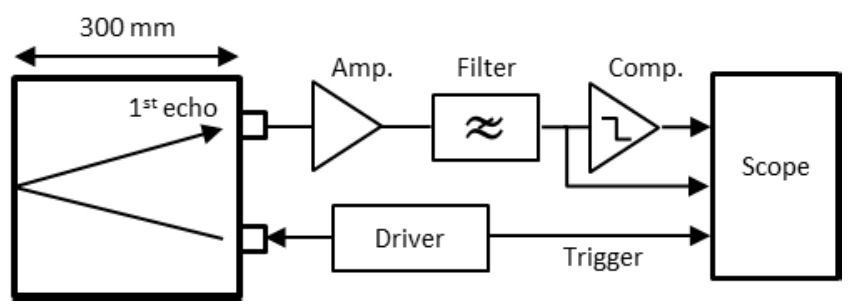

Fig. 8. Experimental setup using ultrasonic transducers. Signals are recorded before and after the comparator and later averaged.

\section{EXPERIMENTAL RESULTS}

Ultrasound signals were recorded before and after a comparator as shown in Fig. 8. Two transducers (Panametrics V106, Olympus, MA 02453, USA) were placed in a pitch-catch configuration on one side of a 300-mm-thick aluminum block that had a cylindrical shape. The edge of the block on the transducers side was rounded to avoid reflections of surface waves. The transmit transducer was connected to a driver, which also triggered an oscilloscope (LeCroy WaveRunner 44Xi). The signal from the receive transducer was amplified and filtered, after which the signal split into two, one cable connecting directly to the scope and the other entering the scope via a comparator (ADCMP600, Analog Devices, Norwood, USA). The scope has an 8-b resolution and a 2-mV minimum sensitivity; the sampling rate was set to $100 \mathrm{MHz}$. The driver, amplifier, and filter are independent units of the pulse-echo system (WaveMaker Duet, custom made for the NDE group of Imperial College London).

The driver was set to transmit a 5-cycle tone-burst with a Hann tapering window and a central frequency of $2 \mathrm{MHz}$. The amplifier gain was set to $60 \mathrm{~dB}$. The response of the band-pass filter in the WaveMaker Duet system is assumed to encompass the tone-burst frequency band and known to have a cut-off frequency below $10 \mathrm{MHz}$. The comparator reference level was calibrated with a potentiometer such that the mean value of the resulting signal at the output was in the middle of the comparator output range; this was to maximize the dynamic input range.

In Fig. 9, signals before and after the comparator (black and gray curves, respectively) are shown. The output of the comparator indicates when the noise is above or below $0 \mathrm{mV}$ in Fig. 9. The shortest time interval between the comparator transitions, i.e., the minimum pulse width, is determined by the comparator and noise bandwidth. The minimum pulse width can be considered equivalent to the effective sampling rate of the binary signal. Its width was found to be below $10 \mathrm{~ns}$, so the effective sampling frequency is greater than $100 \mathrm{MHz}$, which is 50 times greater than the tone-burst central frequency.

Initially, the driver excitation intensity was set such that the receive echoes were just below the noise threshold. The received signals were averaged 500 times. Fig. 10 shows the result of averaging before the comparator stage. The encircle section just before the first echo is known to contain noise due to the receive amplifier only, i.e., there is no mode conversion or any other coherent noise source. This was confirmed by comparing different averaging results where no correlation was found. The noise computed in this interval is used as a reference throughout the experiments. 


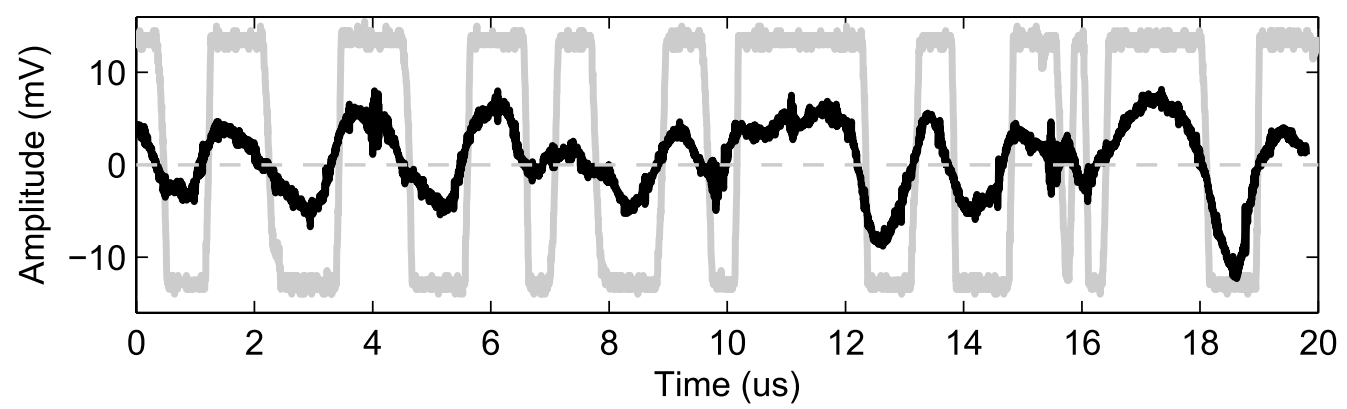

Fig. 9. Comparator input (black curve) and output (gray curve). The output has been normalized to fit the figure.

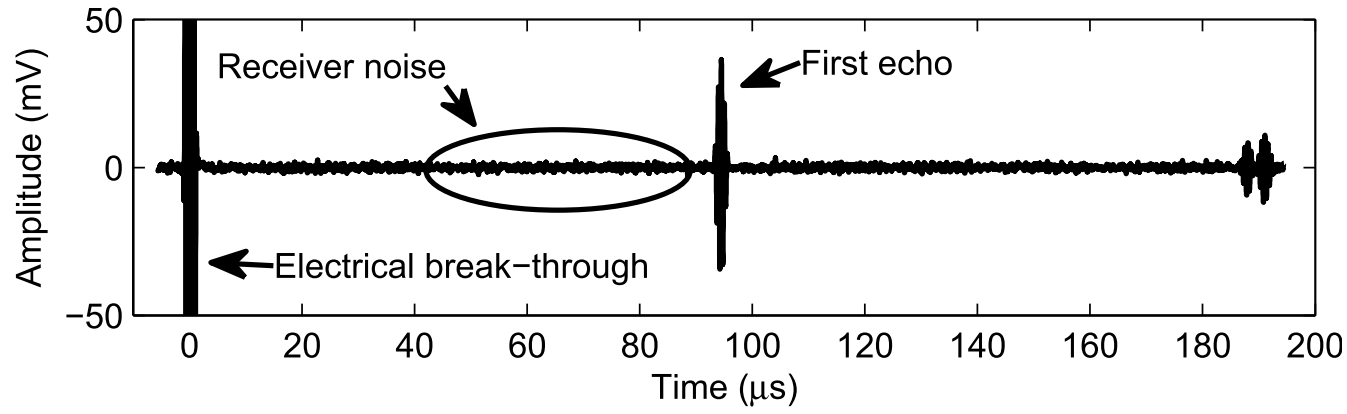

Fig. 10. Receive signal (before comparator) after 500 averages.

Fig. 11(a)-(c) shows the first echo after 500 averages under different excitations and postprocessing conditions. The thick gray trace (8-b) corresponds to the signal before the comparator, the dashed line (1-b) to the signal after the comparator, and the continuous black line (expanded) to the signal after the comparator expanded using (6). Before applying (6), the signal after the comparator was normalized to its maximum value $611.8 \mathrm{mV}$. The output of the expansion operation was limited as indicated in (12) to avoid infinite results.

Fig. 11(a) shows the first echo when the input SNR was $-5.8 \mathrm{~dB}$. This value was estimated by computing the SNR of the signal before the comparator after averaging, which roughly yielded $21.2 \mathrm{~dB}$ - note that 500 averages increase the SNR by roughly $27 \mathrm{~dB}$. The SNR was estimated as the ratio of the maximum value of the signal and the variance of the noise in the interval highlighted in Fig. 10. All the curves in Fig. 11(a) were normalized to their maximum value, and as expected, a good match was found because when the input SNR is below zero, the comparator shows a linear response (after averaging). The SNR difference between the signals before and after the comparator (8-b and 1-b, respectively) was $2.1 \mathrm{~dB}$ as closely predicted by (11) for this low input SNR regime.

The excitation was increased by approximately $13 \mathrm{~dB}$ and the results are shown in Fig. 11(b). The SNR of the 8-b signal was estimated at $33.9 \mathrm{~dB}$ after averaging. The input SNR is roughly $7 \mathrm{~dB}$, which produces a nonlinear response of the comparator as shown by the dashed line. Note that the output of the expansion operation (continuous lines) gives a highly distorted echo. This is due to an imperfect calibration of the comparator. To correct this, the normalized signal after the comparator was scaled by 1.05 and then offset by 0.11 . As a result of this fine-tuning, both the signal before the comparator and after the expansion operation matched very well, which can be appreciated in Fig. 11(c).

However, the input SNR in this case is close to the maximum input SNR ( $\left.\mathrm{SNR}_{\max , \text { in }}\right)$, where saturation starts occurring. Therefore, the SNR after binary quantization deviates from its maximum value (see Fig. 6). The small distortions in the inset of Fig. 11(c) are a consequence of this. Nonetheless, these can be alleviated by filtering out the signal. It is interesting to highlight that the input SNR for this example is very close to the corresponding $\mathrm{SNR}_{\max }$, in for 500 averages [see (18) or Fig. 7]. Hence, if the input SNR is increased any further, saturation will take place and the expansion operation will not prevent severe distortion from occurring.

To conclude the discussion, a visual example of a simulated single copy of the signal before the comparator is shown in Fig. 11(d) (8-b no average); the estimated SNR of this signal is $7 \mathrm{~dB}$. This signal was simulated by adding noise normally distributed with a variance of $27 \mathrm{~dB}$ to the signal before the comparator after 500 averages. By visual inspection, some sections of the signal appear to lie solely on one side of the comparator threshold at zero amplitude, yet binary quantization can recover this signal with low distortion after averaging several repetitions.

Finally, it is important to highlight that in this paper, averaging is used as a means of increasing the SNR after binary acquisition due to its simplicity; however, in many applications, averaging over a large number of realizations may be lengthy and hence impractical. In such cases, the use of pulse compression may be preferred. Pulse compression based on coded sequences can be readily understood as a weighted averaging process, where the aim of using the weights is to accomplish some further postprocessing, such as reducing interference between adjacent bursts. Therefore, 

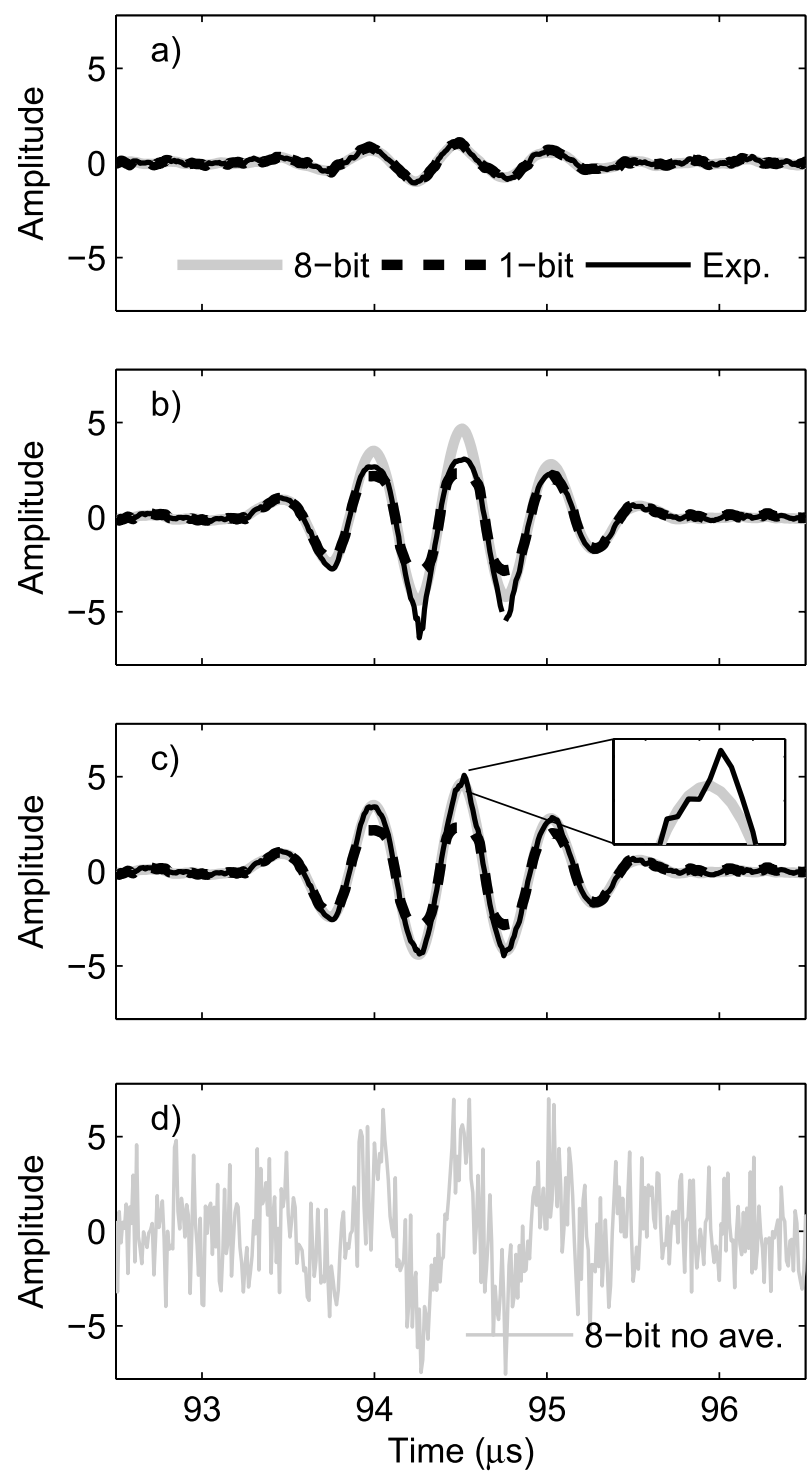

Fig. 11. First echo under different excitations and postprocessing conditions. Thick gray trace $(8-b)$ corresponds to the signal before the comparator, dashed line (1-b) is the signal after the comparator, and the continuous black line (1-b expanded) is the signal after the comparator expanded according to (6); all these traces consist of 500 averages. (a) Input $\mathrm{SNR} \approx-5.8 \mathrm{~dB}$. (b) Input $\mathrm{SNR} \approx 7 \mathrm{~dB}$. (c) Input $\mathrm{SNR} \approx 7 \mathrm{~dB}$ with the comparator output scaled by 1.05 and then offset by 0.11 . (d) Signal with SNR $\approx 7 \mathrm{~dB}$ (8-b no average).

all the results reported for averaging herein apply to coded pulse compression. Any advantage of pulse compression over averaging lies solely on the postprocessing stage and does not directly affect the binary acquisition mechanism here discussed. The analysis of the effect of other pulse compression techniques on binary quantization, e.g., chirp signals, is out of the scope of this paper.

\section{CONCLUSION}

In this paper, the theory of binary quantization of recurrent signals embedded in noise was reviewed in detail. Binary quantization and averaging can be understood as a nonlinear acquisition process similar to standard companding techniques where an expansion function is required to compensate for nonlinearities introduced in the process.
The input SNR where binary quantization is of practical value for ultrasound applications was investigated, and it was found that in most cases, binary quantization can only be employed when the input SNR is below $8 \mathrm{~dB}$. Hence, the input SNR of the binary quantizer is significantly smaller compared with those of standard ADCs, which can be understood as a set of offset binary quantizers. Moreover, the maximum SNR after binary quantization and averaging can be estimated as $10 \log _{10} N-2$; therefore, at least a few hundred of averages are required to produce an SNR at the output greater than $20 \mathrm{~dB}$.

However, the fact that there is only a $2-\mathrm{dB}$ difference between binary quantization and no quantization at all when the signals are below the noise threshold has an important implication in the quantization of signals embedded in noise because standard ADCs can be replaced by comparators and binary latches, and in some cases, even the analog channel may be directly connected to the digital input. All these are especially attractive for applications that require arrays with many channels and high sampling rates, where the sampling rate could be as high as the system clock rate, which in general permits the electronics to be more compact and faster and to consume less energy.

\section{APPENDIX A \\ Variance After AdDing $N$ Realizations of $Q$}

To obtain (8), we first find the variance of $Q$

$$
\sigma_{Q}^{2}=E\left[Q^{2}\right]-E[Q]^{2} .
$$

Note that $E\left[Q^{2}\right]=1$ and $E[Q]$ is given in (4), and hence

$$
\sigma_{Q}^{2}=4 F\left(-\frac{s}{\sigma_{y}}\right)+4 F\left(-\frac{s}{\sigma_{y}}\right)^{2} .
$$

Since $\bar{F}=1-F$ and the sign of $s$ does not affect the result, we can write

$$
\sigma_{Q}^{2}=4 F\left(\frac{s}{\sigma_{y}}\right) \bar{F}\left(\frac{s}{\sigma_{y}}\right) .
$$

Given that the copies of $Q$ are identical and independently distributed (i.i.d.), the variance after adding $N$ copies is $\sigma_{Q, N}^{2}=N \sigma_{Q}^{2}$.

\section{APPENDIX B}

\section{ESTIMATION OF THE STANDARD DEVIATION}

Equation (9) is empirically presented without a proof, and later, its accuracy is corroborated in Section III-B. Nonetheless, the rationale behind this equation for the case $N>c_{N}>0$ is discussed here, which can be readily extended to the remaining case. The need for two cases arises in order to avoid exceeding the domain of (9) during numerical computations. First note that when $N$ is large and $-N<c_{N}<N$, the argument of $F^{-1}$ in (9) can be approximated to

$$
\frac{N-c_{N}-\sigma_{Q, N}}{2 N} \approx 0.5-\frac{E[Q(t)]}{2}-\frac{\sigma_{Q}}{2 \sqrt{N}}
$$

where $\sigma_{Q}^{2}$ is defined in Appendix A. In the case where $F^{-1}$ behaves linearly, for example when $|E[Q(t)]|$ and $\left(\sigma_{Q} / \sqrt{N}\right)$ are smaller than 0.5 , the distribution of the realizations of the ensemble of $Q$ lies mostly within the linear 
regime, and hence (9) can be shown to hold using (6); however, this is not the case in the nonlinear region of $F^{-1}$. Nonetheless, in the nonlinear region, where the ratio $\left(|s| / \sigma_{y}\right)$ increases, $|E[Q(t)]|$ increases, while $\sigma_{Q}$ decreases (see Appendix A), and hence a nonlinear operation over the distribution of the ensemble of $Q$ may still have a quasi-linear impact over its standard deviation within a local interval, as predicted by (9) and later corroborated in Section III-B for the interval of interest.

\section{ACKNOWLEDGMENT}

In order to gain access to supporting information and underlying data, readers are asked to contact the group via http://www.imperial.ac.uk/non-destructive-evaluation. The authors would like to thank Prof. P. Cawley and Prof. R. Challis for the discussions about the topic and suggestions about this paper.

\section{REFERENCES}

[1] K. S. Ho, T. H. Gan, D. R. Billson, and D. A. Hutchins, "Application of pulse compression signal processing techniques to electromagnetic acoustic transducers for noncontact thickness measurements and imaging," Rev. Sci. Instrum., vol. 76, no. 5, p. 054902, 2005.

[2] J. Isla and F. Cegla, "Optimisation of the bias magnetic field of shear wave EMATs," IEEE Trans. Ultrason., Ferroelectr., Freq. Control, to be published, doi: 10.1109/TUFFC.2016.2558467.

[3] I. Payo and J. M. Hale, "Sensitivity analysis of piezoelectric paint sensors made up of PZT ceramic powder and water-based acrylic polymer," Sens. Actuators A, Phys., vol. 168, no. 1, pp. 77-89, 2011.

[4] M. P. Mienkina, C.-S. Friedrich, N. C. Gerhardt, W. G. Wilkening, M. R. Hofmann, and G. Schmitz, "Experimental evaluation of photoacoustic coded excitation using unipolar golay codes," IEEE Trans. Ultrason., Ferroelectr, Freq. Control, vol. 57, no. 7, pp. 1583-1593, Jul. 2010.

[5] T. H. Gan, D. A. Hutchins, D. R. Billson, and D. W. Schindel, "The use of broadband acoustic transducers and pulse-compression techniques for air-coupled ultrasonic imaging," Ultrasonics, vol. 39, no. 3, pp. 181-194, 2001.

[6] M. Ricci, L. Senni, and P. Burrascano, "Exploiting pseudorandom sequences to enhance noise immunity for air-coupled ultrasonic nondestructive testing," IEEE Trans. Instrum. Meas., vol. 61, no. 11, pp. 2905-2915, Nov. 2012.

[7] R. J. Przybyla et al., "In-air rangefinding with an AlN piezoelectric micromachined ultrasound transducer," IEEE Sensors J., vol. 11, no. 11, pp. 2690-2697, Nov. 2011.

[8] D. Hutchins, P. Burrascano, L. Davis, S. Laureti, and M. Ricci, "Coded waveforms for optimised air-coupled ultrasonic nondestructive evaluation," Ultrasonics, vol. 54, no. 7, pp. 1745-1759, 2014.

[9] J. E. Michaels, S. J. Lee, A. J. Croxford, and P. D. Wilcox, "Chirp excitation of ultrasonic guided waves," Ultrasonics, vol. 53, no. 1, pp. 265-270, 2013.

[10] X. Song, D. Ta, and W. Wang, "A base-sequence-modulated Golay code improves the excitation and measurement of ultrasonic guided waves in long bones," IEEE Trans. Ultrason., Ferroelectr., Freq. Control, vol. 59, no. 11, pp. 2580-2583, Nov. 2012.

[11] J. Lin, J. Hua, L. Zeng, and Z. Luo, "Excitation waveform design for Lamb wave pulse compression," IEEE Trans. Ultrason., Ferroelectr. Freq. Control, vol. 63, no. 1, pp. 165-177, Jan. 2016.

[12] M. Ricci, L. Senni, P. Burrascano, R. Borgna, S. Neri, and M. Calderini, "Pulse-compression ultrasonic technique for the inspection of forged steel with high attenuation," Insight-Non-Destruct. Test. Condition Monitor, vol. 54, no. 2, pp. 91-95, 2012.

[13] S. Hirata, M. K. Kurosawa, and T. Katagiri, "Cross-correlation by single-bit signal processing for ultrasonic distance measurement," IEICE Trans. Fundam. Electron., Commun. Comput. Sci., vol. E91-A, no. 4, pp. 1031-1037, 2008

[14] Q. Peng and L.-Q. Zhang, "High-resolution ultrasound displacement measurement using coded excitations," IEEE Trans. Ultrason., Ferroelectr, Freq. Control, vol. 58, no. 1, pp. 122-133, Jan. 2011.

[15] M. Garcia-Rodriguez, Y. Yañez, M. J. Garcia-Hernandez, J. Salazar, A. Turo, and J. A. Chavez, "Application of Golay codes to improve the dynamic range in ultrasonic Lamb waves air-coupled systems," $N D T \& E$ Int., vol. 43, no. 8, pp. 677-686, 2010.
[16] R. E. Challis and V. G. Ivchenko, "Sub-threshold sampling in a correlation-based ultrasonic spectrometer," Meas. Sci. Technol., vol. 22, no. 2, p. 025902, 2011.

[17] R. Challis, V. Ivchenko, and R. Al-Lashi, "Ultrasonic attenuation measurements at very high SNR: Correlation, information theory and performance," J. Phys., Conf. Ser., vol. 457, no. 1, p. 012004, 2013.

[18] L. R. Carley, "An oversampling analog-to-digital converter topology for high-resolution signal acquisition systems," IEEE Trans. Circuits Syst., vol. 34, no. 1, pp. 83-90, Jan. 1987.

[19] P. Carbone and D. Petri, "Effect of additive dither on the resolution of ideal quantizers," IEEE Trans. Instrum. Meas., vol. 43, no. 3, pp. 389-396, Jun. 1994.

[20] R. M. Gray and T. G. Stockham, Jr., "Dithered quantizers," IEEE Trans. Inf. Theory, vol. 39, no. 3, pp. 805-812, May 1993.

[21] A. Derode, A. Tourin, and M. Fink, "Ultrasonic pulse compression with one-bit time reversal through multiple scattering," J. Appl. Phys., vol. 85, no. 9, pp. 6343-6352, 1999.

[22] H. C. Papadopoulos, G. W. Wornell, and A. V. Oppenheim, "Sequential signal encoding from noisy measurements using quantizers with dynamic bias control," IEEE Trans. Inf. Theory, vol. 47, no. 3, pp. 978-1002, Mar. 2001.

[23] A. Ribeiro and G. B. Giannakis, "Bandwidth-constrained distributed estimation for wireless sensor Networks-part I: Gaussian case," IEEE Trans. Signal Process., vol. 54, no. 3, pp. 1131-1143, Mar. 2006.

[24] J. Fang and H. Li, "Distributed adaptive quantization for wireless sensor networks: From delta modulation to maximum likelihood," IEEE Trans. Signal Process., vol. 56, no. 10, pp. 5246-5257, Oct. 2008.

[25] R. M. Gray, "Quantization in task-driven sensing and distributed processing," in Proc. IEEE Int. Conf. Acoust. Speech Signal Process. (ICASSP), vol. 5. May 2006, pp. V-1049-V-1052.

[26] U. S. Kamilov, A. Bourquard, A. Amini, and M. Unser, "One-bit measurements with adaptive thresholds," IEEE Signal Process. Lett., vol. 19 , no. 10, pp. 607-610, Oct. 2012.

[27] E. J. Msechu, S. I. Roumeliotis, A. Ribeiro, and G. B. Giannakis, "Decentralized quantized Kalman filtering with scalable communication cost," IEEE Trans. Signal Process., vol. 56, no. 8, pp. 3727-3741, Aug. 2008.

[28] A. B. Carlson et al., Communication Systems: An Introduction to Signal and Noise in Electrical Communication. New York, NY, USA: McGrawHill, 2002.

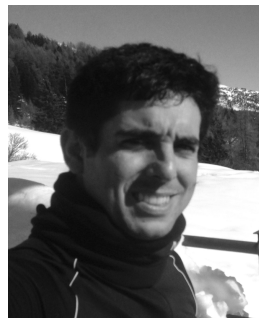

Julio Isla received the Engineering (summa cum laude) degree in telecommunications and electronics and the M.Sc. degree in radio-electronics from the Instituto Superior Polytechnic José Antonio Echeverría (ISPJAE), Havana, Cuba, in 2009 and 2012, respectively. He is currently pursuing the $\mathrm{Ph} . \mathrm{D}$. degree with the Non-Destructive Evaluation Group, Imperial College London, London, U.K.

$\mathrm{He}$ was with the Bioengineering Department, ISPJAE, from 2009 to 2011, and the Institute of Cybernetics, Mathematics and Physics, Havana, from 2011 to 2013. He has been with Permasense Ltd., Horsham, U.K., since 2014, a company that commercializes the low-power electromagnetic acoustic transducer technology that he developed with colleagues. He has also consulted for other international companies. His current research interests include low-power transduction, arrays, instrumentation, and signal processing.

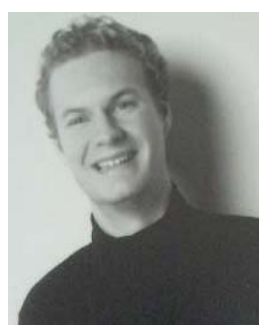

Frederic Celga was born in Freiburg im Breisgau, Germany, in 1980. He received the M.Eng. and Ph.D. degrees in mechanical engineering from Imperial College London, London, U.K., in 2002 and 2006, respectively.

He was a Postdoctoral Research Fellow with the University of Queensland, Brisbane, QLD, Australia. In 2008, he started work as a Lecturer with the Dynamics Section, Mechanical Engineering Department, Imperial College London, and was promoted to Senior Lecturer in 2014. He is a Director of Permasense Ltd., Horsham, U.K., a spin out company that exploits the hightemperature wall thickness monitoring technology that has been developed by him and his colleagues. His current research interests include high temperature ultrasonic monitoring, structural health monitoring, and ultrasonic manipulation of particles and bubbles. 